Proceedings of the 48th International School and Conference on the Physics of Semiconductors "Jaszowiec 2019"

\title{
Secondary Quantization of the Electromagnetic Field in Inhomogeneous Structures Based on Formalism of Scattering Matrix
}

\author{
A.V. Belonovski ${ }^{a, *}$, K.M. Morozov ${ }^{a, b}, K$.A. Ivanov ${ }^{b}$ And M.A. Kaliteevski ${ }^{a, b, c}$ \\ ${ }^{a}$ St-Petersburg Academic University, Khlopina 8/3, 194021 St. Petersburg, Russia \\ ${ }^{b}$ ITMO University, Kronverkskiy pr. 49, 197101 St. Petersburg, Russia \\ ${ }^{c}$ Ioffe Institute, Politekhnicheskaya 26, 194021 St. Petersburg, Russia
}

\begin{abstract}
We show the procedure for the secondary quantization of the electromagnetic field in an inhomogeneous medium, based on the formalism of the scattering matrix. We demonstrate the electromagnetic field profiles, which are obtained as a result of applying the scattering matrix formalism and prove their orthogonality. Finally, we derive the Hamiltonian of the system. The obtained procedure allows us to use more complete representation of quantum theory in a layered medium to describe various effects in layered structures.
\end{abstract}

DOI: 10.12693/APhysPolA.136.649

PACS/topics: 42.50.-p, 42.25.-p

\section{Introduction}

The description of the interaction of radiation with the matter has been the subject of theoretical and experimental research in recent years. It is also one of the important problems in modern physics $[1,2]$.

Ley and Loudon [3] were one of the first researchers who developed a procedure for the secondary quantization of the electromagnetic field in layered structures. In this work, the electromagnetic field is quantized in terms of a continuous set of mode creation and annihilation operators. Later, de Martini et al. [4] used second quantization in inhomogeneous structures to calculate the probability of spontaneous emission in layered structures bounded by semi-infinite media. In these works, the structure of the field modes in the space with the inhomogeneity was assumed to be identical to the mode structure of a homogeneous medium obtained using a periodic $\mathrm{BC}$ (the Born-von Karman boundary condition). In the case of inhomogeneous structures, the approach to the problem of the mode structure of the field based on a periodic BC is not exact and self-consistent, because the presence of an inhomogeneity can lead to significant changes in the mode structure, which is obtained by applying a periodic $\mathrm{BC}$, which in turn can lead to inaccuracies in analysis of systems of finite size.

In Refs. [5-7], the method for quantizing of the electromagnetic field in media containing inhomogeneity (which is proposed to be called $S$-quantization) was developed. The method is based on equating the amplitudes of the wave incident on a quantization box containing inhomogeneity with the amplitudes of the transmitted waves,

*corresponding author; e-mail: leha.s92.92@gmail.com which is equivalent to equating eigenvalues of the scattering matrix ( $S$-matrix) to the unit. Unlike the traditional quantization procedure, based on the periodic boundary conditions, $S$-quantization provides a strict and selfconsistent description of the modes of the electromagnetic field and the density of states.

In this paper, we have demonstrated the electromagnetic field states in a layered medium (for two and three layers), which were obtained using the boundary conditions from papers [5-7] ( $S$-conditions). We also have proved the orthogonal nature of such states and have developed a procedure for the secondary quantization of the electromagnetic field in layered structures by introducing the creation and annihilation operators for the field modes obtained from $S$-conditions.

\section{Model and methodology}

Consider the structure consisting of an arbitrary number of homogeneous layers. Each layer of the medium has a thickness $L_{i}$ and a refractive index $n_{i}$, independent of frequency. Electromagnetic waves incidence arises on the structure to the left and right perpendicular to the layers (Fig. 1).

The general equation for finding the amplitude reflection and transmission coefficients is as follows:

$$
\begin{aligned}
& \left(\begin{array}{c}
t_{1} E_{0}+r_{2} E_{L} \\
E_{L}
\end{array}\right)=\hat{\boldsymbol{M}}\left(n_{N}, L_{N}\right) \prod_{k=N}^{k=2} \hat{\boldsymbol{N}}\left(n_{k-1}, n_{k}\right) \\
& \times \hat{\boldsymbol{M}}\left(n_{k-1}, L_{k-1}\right)\left(\begin{array}{c}
E_{0} \\
r_{1} E_{0}+t_{2} E_{L}
\end{array}\right),
\end{aligned}
$$

where $t_{1}$ and $t_{2}$ are the transmission coefficients for the waves that fall on the left and the right of the structure, respectively. $r_{1}$ and $r_{2}$ are the reflection coefficients for the waves that fall on the left and the right of the structure, respectively. $E_{0}, E_{L}$ are the amplitudes of waves 


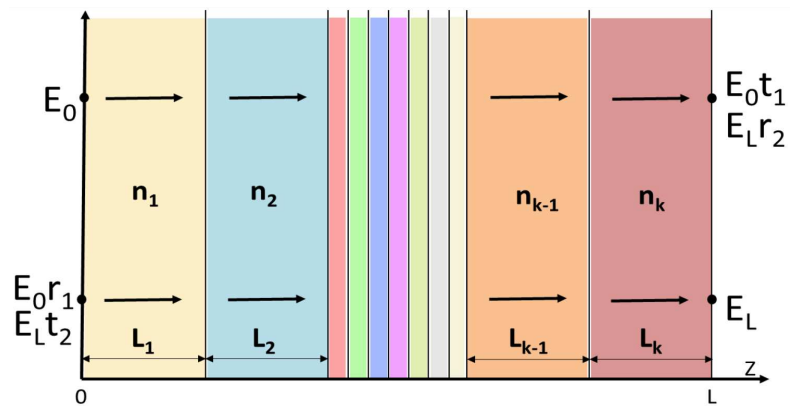

Fig. 1. $N$ layers with the refractive indices $n_{i}$ and thicknesses $L_{i}$. Waves incident on the structure to the left and right perpendicular to the layers.

incident on a structure. The matrix $\hat{M}$ is the homogeneous media transfer matrix, and the matrix $\hat{N}$ is the media transfer matrix

$$
\begin{aligned}
& \hat{M}\left(n_{i}, L_{i}\right)=\left(\begin{array}{cc}
\mathrm{e}^{\mathrm{i} k_{0} n_{i} L_{i}} & 0 \\
0 & \mathrm{e}^{-\mathrm{i} k_{0} n_{i} L_{i}}
\end{array}\right), \\
& \hat{N}\left(n_{i}, n_{j}\right)=\frac{1}{2 n_{j}}\left(\begin{array}{cc}
n_{i}+n_{j} & n_{j}-n_{i} \\
n_{j}-n_{i} & n_{i}+n_{j}
\end{array}\right),
\end{aligned}
$$

where $k_{0}$ is a wave vector. The multiplication of the matrix in (1) occurs in the reverse order, from $k=N$ to $k=2$.

Reference [5] proposes the procedure of quantization of electromagnetic field, based on equating to unity eigenvalues of the scattering matrix of the system, or by equating incoming amplitudes and outgoing amplitudes. $S$-quantization provides an exact and selfconsistent description of modes of the electromagnetic field. The scattering matrix for this structure is as follows:

$$
\left(\begin{array}{c}
E^{+}(L) \\
E^{-}(0)
\end{array}\right)=\left(\begin{array}{ll}
t_{1} & r_{2} \\
r_{1} & t_{2}
\end{array}\right)\left(\begin{array}{c}
E^{+}(0) \\
E^{-}(L)
\end{array}\right)=\hat{\boldsymbol{S}}\left(\begin{array}{c}
E^{+}(0) \\
E^{-}(L)
\end{array}\right) .
$$

Equate the eigenvalues of the scattering matrix to unity

$$
\left(t_{1}-1\right)\left(t_{2}-1\right)-r_{1} r_{2}=0 \text {. }
$$

Using this expression, we can find the spectrum of the eigenfrequencies of the system based on the $S$-condition. The eigenvector of the scattering matrix consists of the complex amplitudes of the fields, incident on the structure

$$
\boldsymbol{B}=\left[1,-\frac{t_{1}-1}{r_{2}}\right] .
$$

The solution of Eq. (5) ensures the spectrum of eigenfrequencies, with which we can calculate the profile of the electromagnetic field of a mode using transfer matrices.

The profile of the electromagnetic field for $\sum_{k=1}^{i-1} L_{k}<z<\sum_{k=1}^{i} L_{k}$ :

$$
\begin{aligned}
& \left(\begin{array}{c}
E^{+}(z) \\
E^{-}(z)
\end{array}\right)=\hat{\boldsymbol{M}}\left(n_{i}, z-\sum_{k=1}^{i-1} L_{k}\right) \prod_{k=2}^{i} \hat{\mathbf{N}}\left(n_{k-1}, n_{k}\right) \\
& \times \hat{\boldsymbol{M}}\left(n_{k-1}, L_{k-1}\right)\left(\begin{array}{c}
E_{0} \\
r_{1} E_{0}+t_{2} E_{L}
\end{array}\right) .
\end{aligned}
$$

\section{Results and discussions}

As the case of one layer coincides with the case of conditions $\mathrm{BC}$, and the cases of many layers are too large in number, we will consider only the cases of two and three layers.

We will consider a structure consisting of two homogeneous layers with refractive indices $n_{1}, n_{2}$, and thicknesses $L_{1}, L_{2}\left(L=L_{1}+L_{2}\right)$, on which the incidence of the electromagnetic wave takes place on the left and right, perpendicular to the layers. From Eq. (1) we get the values $t_{1}, t_{2}, r_{1}, r_{2}$ :

$$
\begin{aligned}
& \left\{\begin{array}{l}
r_{1}=\mathrm{e}^{2 \mathrm{i} k_{0} n_{1} L_{1}} r_{12}, \\
r_{2}=\mathrm{e}^{2 \mathrm{i} k_{0} n_{2} L_{2}} r_{21},
\end{array}\right. \\
& \left\{\begin{array}{l}
t_{1}=\mathrm{e}^{\mathrm{i} k_{0} n_{1} L_{1}} \mathrm{e}^{\mathrm{i} k_{0} n_{2} L_{2}} t_{12}, \\
t_{2}=\mathrm{e}^{\mathrm{i} k_{0} n_{1} L_{1}} \mathrm{e}^{\mathrm{i} k_{0} n_{2} L_{2}} t_{21},
\end{array}\right.
\end{aligned}
$$

where $r_{12}=\frac{n_{1}-n_{2}}{n_{1}+n_{2}}, t_{12}=\frac{2 n_{1}}{n_{1}+n_{2}}, r_{21}=-r_{12}$, $t_{21}=2-t_{12}$. Substituting these values into the equation for the eigenfrequencies (5), we obtain the values of the wave vector

$$
k_{0}=\frac{2 \pi N}{n_{1} L_{1}+n_{2} L_{2}}, \quad N \in \mathbb{Z} .
$$

From this expression, we find the spectrum of eigenfrequencies. The eigenvector for this case

$$
\boldsymbol{B}=\left[1, \mathrm{e}^{2 \mathrm{i} k_{0} n_{1} L_{1}}\right] .
$$

Orthogonality for the profiles of the electromagnetic field of modes is found (Ref. [3]) from the expression

$$
\begin{aligned}
& \int_{0}^{L} n^{2} E_{N}(z) E_{N^{\prime}}^{*}(z) \mathrm{d} z= \\
& \quad \int_{0}^{L} n^{2}\left(E_{N}^{+}(z)+E_{N}^{-}(z)\right)\left(E_{N}^{+}(z)+E_{N}^{-}(z)\right)^{*} \mathrm{~d} z .
\end{aligned}
$$

Substituting the field values from (7) and the values from (8-11), we get

$\int_{0}^{L} n^{2} E_{N}(z) E_{N^{\prime}}^{*}(z) \mathrm{d} z=2 E_{0}^{2}\left(n_{1}^{2} L_{1}+n_{2}^{2} L_{2}\right) \delta_{N, N^{\prime}}$

Thus, it is clear that the profiles are orthogonal to each other.

Next we will consider the structure that consists of three layers. For the simplicity of formulae, we introduce additional conditions: $n_{1} L_{1}=n_{2} L_{2}=n_{3} L_{3}$, and $n_{1}=n_{3}$. We introduce the notation $k_{0} n_{1} L_{1}=\alpha$. From Eq. (1) we get the values $t_{1}, t_{2}, r_{1}, r_{2}$ :

$$
\begin{aligned}
& r_{1}=r_{2}=r=\mathrm{e}^{2 \mathrm{i} \alpha} \frac{r_{12}-r_{12} \mathrm{e}^{2 \mathrm{i} \alpha}}{1-r_{12}^{2} \mathrm{e}^{2 \mathrm{i} \alpha}}, \\
& t_{1}=t_{2}=t=\mathrm{e}^{3 \mathrm{i} \alpha} \frac{t_{12} t_{21}}{1-r_{12}^{2} \mathrm{e}^{2 \mathrm{i} \alpha}} .
\end{aligned}
$$

If we substitute values into the equation for the eigenfrequencies (5), we obtain the values of $\mathrm{e}^{\mathrm{i} \alpha}$ : 


$$
\left\{\begin{array}{l}
\mathrm{e}^{\mathrm{i} \alpha_{1}}=1, \\
\mathrm{e}^{\mathrm{i} \alpha_{2}}=-\frac{t_{12}}{2} \pm \sqrt{\left(\frac{t_{12}}{2}\right)^{2}-1}, \\
\mathrm{e}^{\mathrm{i} \alpha_{3}}=-\frac{t_{21}}{2} \pm \sqrt{\left(\frac{t_{21}}{2}\right)^{2}-1} .
\end{array}\right.
$$

We got three kinds of solutions (denoted by indices). From this expression, we find the spectrum of eigenfrequencies. The eigenvector for the case $\alpha_{2}: \boldsymbol{B}=[1,1]$, for the case $\alpha_{3}: \boldsymbol{B}=[1,-1]$. Case $\alpha_{1}$ coincides with the solution with the $\mathrm{BC}$. The eigenvector can be the same as for condition $\alpha_{2}$ or $\alpha_{3}$. Orthogonality for the profiles of the electromagnetic field of modes is found from the expression (12).

For $\alpha_{1}(\boldsymbol{B}=[1,1])$ :

$$
\begin{aligned}
& \int_{0}^{L} n^{2} E_{N}(z) E_{N^{\prime}}^{*}(z) \mathrm{d} z= \\
& \quad E_{0}^{2}\left(n_{1}^{2} L_{1}+n_{2}^{2} L_{2}+n_{3}^{2} L_{3}\right) \delta_{N, N^{\prime}} .
\end{aligned}
$$

For $\alpha_{1}(\boldsymbol{B}=[1,-1])$

$$
\begin{aligned}
& \int_{0}^{L} n^{2} E_{N}(z) E_{N^{\prime}}^{*}(z) \mathrm{d} z= \\
& \quad E_{0}^{2}\left(n_{1}^{2} L_{1}+n_{1}^{2} L_{2}+n_{3}^{2} L_{3}\right) \delta_{N, N^{\prime}} .
\end{aligned}
$$

For $\alpha_{2}$ :

$$
\begin{aligned}
& \int_{0}^{L} n^{2} E_{N}(z) E_{N^{\prime}}^{*}(z) \mathrm{d} z= \\
& \quad E_{0}^{2}\left(n_{1}^{2} L_{1}+n_{1} n_{2} t_{12} L_{2}+n_{3}^{2} L_{3}\right) \delta_{N, N^{\prime}} .
\end{aligned}
$$

For $\alpha_{3}$ :

$$
\begin{aligned}
& \int_{0}^{L} n^{2} E_{N}(z) E_{N^{\prime}}^{*}(z) \mathrm{d} z= \\
& \quad E_{0}^{2}\left(n_{1}^{2} L_{1}+n_{1} n_{2} t_{21} L_{2}+n_{3}^{2} L_{3}\right) \delta_{N, N^{\prime}} .
\end{aligned}
$$

The different values before $\delta_{N, N^{\prime}}$ found in (17-20) will affect the normalization coefficient. The electromagnetic field is normalized so that the integral of the energy density of the electromagnetic field of the mode over the "quantization box" associated with the energy of zero-point vibrations of the mode [8]:

$$
\frac{2}{4 \pi} \int_{0}^{L} n^{2} E_{N}(z) E_{N}^{*}(z) \mathrm{d} z=\frac{\hbar w}{2} .
$$

Here it is easy to calculate the normalization factor

$$
E_{0}=\sqrt{\frac{\pi \hbar w_{k}}{V_{k}}},
$$

where $V_{k}=n_{1}^{2} L_{1}+n_{2}^{2} L_{2}$ for two-layer structure, and $V_{k}=n_{1}^{2} L_{1}+n_{2}^{2} L_{2}+n_{3}^{2} L_{3}$ for three-layer structure for case $\alpha_{1} \boldsymbol{B}=[1,1]$ ) etc. Now, substituting (22) into (1) and (17-20), we obtain the classical form for orthogonality

$$
\int_{0}^{L} n E_{N}(z) E_{N^{\prime}}^{*}(z) \mathrm{d} z=2 \pi \hbar w \delta_{N, N^{\prime}} .
$$

The electromagnetic field is quantized by introducing the operators of the creation and annihilation of modes. The creation and annihilation operators for standing waves are $\hat{a}_{k}^{+}$and $\hat{a}_{k}$. The following commutation relations hold for them:

$$
\left[\hat{a}_{k}, \hat{a}_{k^{\prime}}^{+}\right]=\delta_{k, k^{\prime}} \text {. }
$$

When quantizing only in the $X Y$ plane and with one linear polarization, the usual quantization procedure of the electromagnetic field is given by the electromagnetic field operator [3]:

$$
\hat{E}^{+}(z, t)=\mathrm{i} \int_{0}^{\infty} \hat{a}_{k} E_{k} \mathrm{e}^{-\mathrm{i} w_{k} t} \mathrm{~d} k .
$$

The Hamiltonian can be written as

$$
\hat{H}=2 \int_{0}^{L} \mathrm{~d} z \frac{1}{4 \pi} n^{2}(z) \hat{E}^{-}(z, t) \hat{E}^{+}(z, t),
$$

and this reduces with the use of the orthogonality relations (23) and the commutation relations for operators (24) to the expected form

$$
\hat{H}=\int_{0}^{\infty} \hbar w_{k} \hat{a}_{k}^{+} \hat{a}_{k} \mathrm{~d} k .
$$

The resulting procedure of secondary quantization complements and generalizes the quantum-optical description of the electromagnetic field in a layered structure, which was developed earlier, particularly in [1-7]. The expressions obtained here will allow us to use a more complete representation of quantum theory in a layered medium to describe various effects in layered structures.

\section{Conclusion}

In this paper, we have derived the form of the profiles of the eigenmodes of the electromagnetic field in a layered structure, which is obtained as a result of applying $S$-quantization. We have proved orthogonality of these eigenmodes of a layered structure and developed a procedure for the secondary quantization of the electromagnetic field in layered structures, based on $S$-quantization. This representation provides a rigorous and self-consistent description of various parameters, in particular, electromagnetic field profiles, the density of state, and probability of spontaneous emission for arbitrary layered structures.

\section{Acknowledgments}

This work has been supported by Russian Science Foundation (Project No. 16-12-10503).

\section{References}

[1] V.L. Ginzburg, Usp. Fiz. Nauk 140, 687 (1983).

[2] R. Loudon, Quantum Theory of Light, Oxford University Press, Oxford 2000. 
[3] M. Ley, R. Loudon, J. Mod. Opt. 34, 227 (1987).

[4] F. de Martini, M. Marrocco, P. Mataloni, L. Crescentini, R. Loudon, Phys. Rev. A 43, 2480 (1991).

[5] M.A. Kaliteevski, V.A. Mazlin, K.A. Ivanov, A.R. Gubaydullin, Opt. Spectrosc. 119, 832 (2015).

[6] M.A. Kaliteevskii, A.R. Gubaydullin, K.A. Ivanov, V.A. Mazlin, Opt. Spectrosc. 121, 410 (2016).
[7] K.A. Ivanov, A.R. Gubaidullin, K.M. Morozov, M.E. Sasin, M.A. Kaliteevskii, Opt. Spectrosc. 122 835 (2017).

[8] R. Coccioli, M. Boroditsky, K.W. Kim, Y. RahmatSamii, E. Yablonovitch, Proc. IEEE Optoelectron. 145, 391 (1998). 